\title{
BCS to Bose Crossover in Anisotropic Superconductors.
}

\author{
J.P.Wallington and James F.Annett. \\ University of Bristol, H.H. Wills Physics Laboratory, \\ Royal Fort, Tyndall Ave, \\ Bristol BS8 1TL, United Kingdom.
}

(April 21, 2018)

\begin{abstract}
In this work we use functional integral techniques to examine the nearest neighbour attractive Hubbard model on a quasi-2D lattice. It is a simple phenomenological model for the high- $\mathrm{T}_{c}$ cuprates that allows both extended (non-local) $s$ - and $d$-wave singlet superconductivity as well as mixed symmetry states. The Hartree-Gor'kov mean field theory of the model has a finite temperature phase diagram which shows a transition from pure $s$-wave to pure $d$-wave superconductivity, via a mixed symmetry $s+i d$ state, as a function of doping. Including Gaussian fluctuations we examine the crossover from weak-coupling BCS superconductivity to the strong-coupling Bose-Einstein condensation of composite $s$ - or $d$-wave bosons and comment on the origin and symmetry of the pseudogap.
\end{abstract}

\section{INTRODUCTION}

The physics of the high- $T_{c}$ cuprates is unusual and fascinating for a number of reasons. In this work we explore two of them: the symmetry of the superconducting pairing state and the existence of the pseudogap state between the insulator and the superconductor.

In recent years the experimental evidence has become overwhelming that the cuprate high- $\mathrm{T}_{c}$ superconductors possess an anisotropic $d$-wave pairing state [1]. Angle resolved photoemission (ARPES) experiments [2,3] and measurements of the temperature dependence of the penetration depth 4 - 6 both strongly support this scenario, but perhaps the most convincing evidence comes from the elegant measurements of Wollman et al. [7] and of Tsuei et al. [8] since they do not depend on the microscopic physics of the energy gap, but instead depend only on the order parameter phase.

Whilst there is a consensus on the $d$-wave nature of the dominant part of the order parameter, there is continuing controversy over the possible existence of a sub-dominant $s$-wave contribution [9, 10]. The existence, or otherwise, of a subdominant $s$-wave component has profound consequences for the nature of the microscopic pairing mechanism. For example, antiferromagnetic spin fluctuations lead to attraction in the $d_{x^{2}-y^{2}}$ pairing channel, but are pair breaking in the $s$-wave channel 11. Similarly the Hubbard model with a positive (repulsive) on-site interaction U may have a $d_{x^{2}-y^{2}}$ paired ground state, but would presumably not support $s$-wave Cooper pairs. In the case of $\mathrm{YBa}_{2} \mathrm{Cu}_{3} \mathrm{O}_{7}$ the orthorhombic crystal symmetry makes some non-zero $s$-wave component inevitable, but a large $s$-wave component would be difficult to reconcile with either of these pairing mechanisms. On the other hand, pairing mechanisms based on electron-phonon interactions, polarons, or other non-magnetic excitations (e.g. excitons, acoustic plasmons) could be compatible with either $s$-wave or $d$-wave pairing states [12]. In these models, whether $s$-wave, $d$-wave or mixed pairs are more strongly favoured would depend on details of the model parameters, and could even vary from compound to compound. Indeed there is some evidence that the n-type cuprate superconductors are $s$-wave 13 (or at least they have no zeros in the gap $|\Delta(\mathbf{k})|$ on the Fermi surface). This would imply that either the pairing mechanism is different for the $\mathrm{n}$ - and $\mathrm{p}$-type materials, or that the mechanism allows both $s$-wave or $d$-wave ground states depending on the band filling.

The second remarkable feature of the cuprates is the "pseudogap" occuring in underdoped materials. Transport experiments [14], ARPES [2, 3], NMR [15, 16], and optical conductivity 17, 18] all detect a loss of spectral weight at low energies in the one-particle excitation spectrum below a certain temperature, $\mathrm{T}^{*}>\mathrm{T}_{c}$. Furthermore, the k-specific nature of the ARPES technique has shown that the pseudogap has nodes at the same places as the superconducting gap. These data and the fact that the coherence length is known to be very small in the cuprates $(\xi(0) \simeq 10-20 \AA$ in the $\mathrm{CuO}_{2}$ planes) points to a strong-coupling scenario in which superconducting fluctuations play a major role.

Although there is as yet no concensus on the exact nature of the fluctuations, it is generally believed that the opening of the pseudogap at $\mathrm{T}^{*}$ is due to the formation of an incoherent pairing state above $\mathrm{T}_{c}$.

Two competing scenarios have emerged to explain this phenomenon. In the first, exemplified by the work of Emery and Kivelson [19], the relevant fluctuations are in the phase. The stiffness to the phase fluctuations is $n_{s}(0) / m^{*}$ 
(where $n_{s}(0)$ is the zero temperature superfluid density and $m^{*}$ is the effective quasiparticle mass), and where this is small, such as in the underdoped cuprates, the true $\mathrm{T}_{c}$, where phase coherence is established, can be well below the mean field transition temperature which is identified with $\mathrm{T}^{*}$.

The second scenario is best illustrated by the works of Nozières and Schmitt-Rink 20] (which predates the discovery of the high- $\mathrm{T}_{c}$ compounds), Dreschler and Zwerger [21] and Sá de Melo, Randeria and Engelbrecht [22] (see Ref. [23] for a review). By considering both magnitude and phase fluctuations in $s$-wave superconductors in the vicinity of the critical temperature, these authors examined the crossover from BCS-like superconductivity of weakly bound pairs to the Bose condensation of tightly bound pairs as $U$, the strength of the interaction, was varied. The results are summarised in Fig. 1. For weak pairing the fluctuating and mean field theories are essentially the same. However as $U$ is increased large differences become apparent: whilst the mean field critical temperature, $\mathrm{T}_{c}^{M F}$, keeps getting larger, the effects of fluctuations cause the true $\mathrm{T}_{c}$ to flatten off, for the continuum model, or even to decrease in the case of the lattice model. The difference between $\mathrm{T}_{c}^{M F}$ and $\mathrm{T}_{c}$ gives an estimate for the regime where fluctuations dominate and may correspond to the pseudogap regime in which bosonic pairs exist but are not condensed into a superfluid.

In this paper we will address both the pairing symmetry and the fluctuation issues in a systematic way. We broadly follow the functional integral approach taken by Dreschler and Zwerger [21] and Sá de Melo, Randeria and Engelbrecht [22], but explicitly including $d$-wave pairing. In section II we introduce the attractive nearest neighbour Hubbard model as a simple phenomenological model of the cuprates and derive from it a Landau-Ginzburg-Wilson (LGW) functional describing $s$ - and $d$-wave superconducting fields. In section III we examine the BSC-like mean field theory of the model by finding the saddle point solutions of the LGW functional which show $s$ - and $d$-wave and mixed symmetry phases. Finally, in section IV, we include Gaussian fluctuations about the saddle point in order to study the strong coupling bosonic limit. This limit is especially interesting in this model because of the competition between preformed pairs of different symmetries. We also comment on the symmetry and origin of the pseudogap.

\section{LANDAU-GINZBURG-WILSON THEORY}

To investigate the symmetry of the order parameter and the nature of the bosonic limit for anisotropic pairs, we consider a simple phenomenological model of the cuprates, the attractive nearest neighbour Hubbard model on a 2D square lattice. It is the simplest model that allows $s$-wave, $d$-wave or mixed symmetry pairing states to occur. Its Hamiltonian is,

$$
\hat{H}=\hat{H}_{0}+\hat{H}_{I},
$$

where $\hat{H}_{0}$ contains the chemical potential and a nearest neighbour hopping term. The interaction Hamiltonian describes an electron-electron attraction on nearest neighbour sites:

$$
\hat{H}_{I}=-V \sum_{\langle i j\rangle} \hat{n}_{i} \hat{n}_{j},
$$

where $\sum_{\langle i j\rangle}$ denotes summation over all of the bonds between the nearest neighbour sites. For the sake of simplicity, we do not include an on-site interaction term.

In order to investigate the thermodynamics of this model it is sufficient to consider the partition function. In the coherent state functional integral representation it is,

$$
\begin{aligned}
& \mathcal{Z}=\int \mathcal{D}\left[c^{\dagger}, c\right] e^{\mathcal{S}}, \\
& \mathcal{S}=\int_{0}^{\beta} d \tau\left[\sum_{i \sigma} c_{i \sigma}^{\dagger}(\tau)\left(-\partial_{\tau}\right) c_{i \sigma}(\tau)-H\left(c^{\dagger}, c\right)\right],
\end{aligned}
$$

where $c$ and $c^{\dagger}$ are the Grassmannian counterparts to the Fermi operators $\hat{c}$ and $\hat{c}^{\dagger}$.

Unfortunately, the interaction part of the Hamiltonian is quartic in the Grassmann (fermion) fields so the functional integral cannot be evaluated exactly. However, by using the Hubbard-Stratonovič transformation [24.25] the quartic problem of interacting electrons can be converted to the more tractable quadratic problem of non-interacting electrons coupled to a random (Gaussian weighted) Bose field. In order to make this transformation, the electronic Hamiltonian must first be decomposed into a product of fermionic bilinears. This can be done in a number of different ways, but the purpose of this paper is to study superconductivity so we choose what might be called the Gor'kov decomposition in terms of pairing operators: 


$$
\hat{H}_{I}=-V \sum_{\langle i j\rangle} \operatorname{tr}\left(\hat{F}_{i j}^{\dagger} \hat{F}_{i j}\right)
$$

with the pairing matrix written as,

$$
\hat{F}_{i j}=\frac{i \sigma_{y}}{\sqrt{2}}\left(\hat{B}_{i j}-\boldsymbol{\sigma} \cdot \hat{\mathbf{B}}_{i j}\right)
$$

where $\boldsymbol{\sigma}$ is the vector of Pauli matrices, and

$$
\begin{aligned}
\hat{B}_{i j} & =\frac{1}{\sqrt{2}}\left(\hat{c}_{j \uparrow} \hat{c}_{i \downarrow}-\hat{c}_{j \downarrow} \hat{c}_{i \uparrow}\right), \\
\hat{\mathbf{B}}_{i j} & =\left\{\begin{array}{l}
\frac{1}{\sqrt{2}}\left(\hat{c}_{j \downarrow} \hat{c}_{i \downarrow}-\hat{c}_{j \uparrow} \hat{c}_{i \uparrow}\right), \\
\frac{i}{\sqrt{2}}\left(\hat{c}_{j \downarrow} \hat{c}_{i \downarrow}+\hat{c}_{j \uparrow} \hat{c}_{i \uparrow}\right), \\
\frac{1}{\sqrt{2}}\left(\hat{c}_{j \uparrow} \hat{c}_{i \downarrow}+\hat{c}_{j \downarrow} \hat{c}_{i \uparrow}\right) .
\end{array}\right.
\end{aligned}
$$

The scalars, $\hat{B}_{i j}$, are even under both time reversal and parity whilst the vectors, $\hat{\mathbf{B}}_{i j}$, are odd under both. In the manner of Volovik and Gor'kov [26], we interpret $\hat{B}_{i j}$ and $\hat{\mathbf{B}}_{i j}$ as the annihilation operators for singlet and triplet Cooper pairs on the bonds $\langle i j\rangle$. In terms of these operators the interaction Hamiltonian becomes:

$$
\hat{H}_{I}=-V \sum_{<i j>}\left(\hat{B}_{i j}^{\dagger} \hat{B}_{i j}+\hat{\mathbf{B}}_{i j}^{\dagger} \cdot \hat{\mathbf{B}}_{i j}\right)
$$

where we have used $\operatorname{tr}\left(\sigma_{i}\right)=0$ and $\operatorname{tr}\left(\sigma_{i} \sigma_{j}\right)=2 \delta_{i j}$.

Although it would be interesting to study triplet superconductivity, especially in light of the exciting recent discovery 27] of $p$-wave pairing in strontium ruthenate, $\mathrm{Sr}_{2} \mathrm{RuO}_{4}$, the focus of this work is on anisotropic singlet pairing as seen in the high- $\mathrm{T}_{c}$ cuprates. Therefore, for the sake of simplicity, we restrict our attention to the singlet fields.

Having decomposed the interaction Hamiltonian into a product of fermionic bilinears we make the HubbardStratonovič transformation. Tensor valued Bose fields, $\Delta_{i j}$, are introduced conjugate to the fermion bilinears, $\hat{B}_{i j}$. (Had we kept the triplet operators we would have needed to introduce an additional vector of triplet pairing fields, $\left.\boldsymbol{\Delta}_{i j}.\right)$ In terms of these the partition function becomes,

$$
\begin{aligned}
& \mathcal{Z}=\int \mathcal{D}\left[\Delta^{*}, \Delta\right] e^{S}, \\
& S=-V \int_{0}^{\beta} \mathrm{d} \tau \sum_{\langle i j\rangle}\left|\Delta_{i j}(\tau)\right|^{2}+\ln \int \mathcal{D}\left[c^{\dagger}, c\right] e^{\mathcal{S}_{f}},
\end{aligned}
$$

with the linearised fermions contained in,

$$
\begin{aligned}
\mathcal{S}_{f}=\int_{0}^{\beta} \mathrm{d} \tau & {\left[\sum_{i} c_{i}^{\dagger}(\tau)\left(-\partial_{\tau}\right) c_{i}(\tau)-H_{0}\left(c^{\dagger}, c\right)\right.} \\
& \left.-V \sum_{\langle i j\rangle}\left(\Delta_{i j}^{*}(\tau) B_{i j}(\tau)+\Delta_{i j}(\tau) B_{i j}^{\dagger}(\tau)\right)\right] .
\end{aligned}
$$

The Hubbard-Stratonovič fields, $\Delta_{i j}$, are defined on each bond $\langle i j\rangle$ of the lattice. However, it is generally more convenient to work with site-centred combinations possessing a definite symmetry. On a square lattice, every site, $i$, has two fields associated with it, one in the $x$-direction and one in the $y$-direction. (We could associate four fields with each point but that introduces problems with double counting.) Rather than talk about $x$ - and $y$-bond fields, it is natural to form linear combinations of them:

$$
\begin{aligned}
\Delta_{s} & =\frac{1}{2}\left(\Delta_{x}+\Delta_{y}\right), \\
\Delta_{d} & =\frac{1}{2}\left(\Delta_{x}-\Delta_{y}\right),
\end{aligned}
$$

where $\Delta_{a}\left(\mathbf{r}_{i}\right)=\Delta_{i i+a}$ for $a=x, y$ according to the direction of the bond $\langle i j\rangle$. We call these the $s$ - and $d$-wave fields because, in the spatially homogeneous limit, they become the order parameters for (extended) $s$ - and $d$-wave 
superconductivity. Unlike the $x$ and $y$ fields, the $s$ and $d$ order parameters transform as irreducible representations of the tetragonal crystallographic point group, $D_{4 h}$. In the notation of Ref. [28] they belong to the $A_{1 g}$ and $B_{1 g}$ representations of $D_{4 h}$. The $d$-wave representation, $B_{1 g}$, corresponds to an order parameter with $x^{2}-y^{2}$ symmetry as seen in the cuprate materials.

Proceeding with the calculation, the action is diagonalised by the introduction of Fourier transformed fields:

$$
\begin{aligned}
c(k) & =\frac{1}{\sqrt{\beta N}} \int_{0}^{\beta} \mathrm{d} \tau \sum_{i} c_{i}(\tau) e^{i \omega_{n} \tau} e^{i \mathbf{k} \cdot \mathbf{r}_{i}}, \\
\Delta_{\alpha}(q) & =\frac{1}{\sqrt{\beta N}} \int_{0}^{\beta} \mathrm{d} \tau \sum_{i} \Delta_{\alpha}\left(\mathbf{r}_{i}, \tau\right) e^{i \omega_{\nu} \tau} e^{i \mathbf{q} \cdot \mathbf{r}_{i}}
\end{aligned}
$$

in which $k=\left(i \omega_{n}, \mathbf{k}\right)\left[q=\left(i \omega_{\nu}, \mathbf{q}\right)\right]$ where the fermion [boson] Matsubara frequencies are $\omega_{n}=(2 n+1) \frac{\pi}{\beta}\left[\omega_{\nu}=(2 \nu) \frac{\pi}{\beta}\right]$.

It is then a straightforward matter to integrate out the vestigial fermionic degrees of freedom. The purely bosonic action then reads

$$
\mathcal{S}=\mathcal{S}_{0}+\mathcal{S}_{b}
$$

where the non-interacting and interacting terms are respectively,

$$
\begin{aligned}
& \mathcal{S}_{0}=-\operatorname{Tr} \ln \left(\mathbf{G}_{0}\right), \\
& \mathcal{S}_{b}=-\frac{V}{2} \sum_{q, \alpha}\left|\Delta_{\alpha}(q)\right|^{2}+\operatorname{Tr} \ln \left(\mathbf{1}-\mathbf{V G}_{0}\right) .
\end{aligned}
$$

The first term, $\mathcal{S}_{0}$, corresponding to the free electron action depends only on the Nambu Green's function matrix,

$$
\mathbf{G}_{0}\left(k, k^{\prime}\right)=\left(\begin{array}{cc}
G_{0}(k) & 0 \\
0 & -G_{0}^{*}(k)
\end{array}\right) \delta_{k k^{\prime}}
$$

in which $G_{0}(k)=\left(i \omega_{n}-\xi_{\mathbf{k}}\right)^{-1}$ is the free fermion propagator, and $\xi_{\mathbf{k}}=\epsilon_{\mathbf{k}}-\mu$. For nearest neighbour hopping the band-energy is $\epsilon_{\mathbf{k}}=-2 t \sum_{i=x, y} \cos \left(k_{i}\right)$.

The second term, $\mathcal{S}_{b}$, which describes the effects of electron-electron interaction, depends not only on the electron Green's function but also on the bosonic off-diagonal potential matrix,

$$
\mathbf{V}\left(k, k^{\prime}\right)=\frac{V}{\sqrt{2 \beta N}}\left(\begin{array}{cc}
0 & \Delta_{\alpha}\left(k-k^{\prime}\right) \\
\Delta_{\alpha}^{*}\left(-k+k^{\prime}\right) & 0
\end{array}\right) \zeta_{\mathbf{k}, \mathbf{k}^{\prime}}^{\alpha},
$$

where the Einstein summation convention has been used for repeated Greek indices and,

$$
\zeta_{\mathbf{k}, \mathbf{k}^{\prime}}^{\alpha}=\frac{1}{2}\left(e^{i k_{x}}+e^{-i k_{x}^{\prime}}\right) \pm \frac{1}{2}\left(e^{i k_{y}}+e^{-i k_{y}^{\prime}}\right), \quad \alpha=s, d
$$

In the limit that $\mathbf{k}=\mathbf{k}^{\prime}$ these become,

$$
\zeta^{\alpha}(\mathbf{k})=\zeta_{\mathbf{k}, \mathbf{k}}^{\alpha}= \begin{cases}\cos \left(k_{x}\right)+\cos \left(k_{y}\right) & \alpha=s \\ \cos \left(k_{x}\right)-\cos \left(k_{y}\right) & \alpha=d\end{cases}
$$

which reflect the point group symmetries of the order parameters.

Expanding the logarithm in $\mathcal{S}_{b}$ as a power series to fourth order, only the second and fourth order terms are non-zero. They are shown diagrammatically in Fig. 3

After a trivial rescaling of $\Delta$ by $V^{\frac{1}{2}}$, the bosonic action to second order reads,

$$
\mathcal{S}_{b}^{(2)}=-\frac{1}{2} \sum_{q}\left(\delta^{\alpha \beta}-V \chi_{0}^{\alpha \beta}(q)\right) \Delta_{\alpha}^{*}(q) \Delta_{\beta}(q),
$$

where the particle-particle susceptibilities are,

$$
\begin{aligned}
\chi_{0}^{\alpha \beta}(q) & =+\frac{1}{\beta N} \sum_{k} G_{0}(k) G_{0}^{*}(k+q) \zeta_{\mathbf{k}, \mathbf{k}+\mathbf{q}}^{\alpha} \zeta_{\mathbf{k}+\mathbf{q}, \mathbf{k}}^{\beta}, \\
& =-\frac{1}{N} \sum_{\mathbf{k}}\left[\frac{n_{f}\left(\xi_{\mathbf{k}+\frac{\mathbf{q}}{2}}\right)+n_{f}\left(\xi_{\mathbf{k}-\frac{\mathbf{q}}{2}}\right)-1}{i \omega_{\nu}+\xi_{\mathbf{k}+\frac{\mathbf{q}}{2}}+\xi_{\mathbf{k}-\frac{\mathbf{q}}{2}}}\right] \zeta_{\mathbf{k}-\frac{\mathbf{q}}{2}, \mathbf{k}+\frac{\mathbf{q}}{2}} \zeta_{\mathbf{k}+\frac{\mathbf{q}}{2}, \mathbf{k}-\frac{\mathbf{q}}{2}}^{\beta},
\end{aligned}
$$


where $n_{f}(x) \equiv\left[e^{\beta x}+1\right]^{-1}$ is the Fermi function.

Regardless of the flavour indices, $\chi_{0}^{\alpha \beta}(q)$ is a maximum at zero frequency and zero momentum. Keeping only relevant terms, expansion of the Gaussian action about $q=0$ gives,

$$
\mathcal{S}_{b}^{(2)}=-\frac{1}{2} \sum_{q}\left(r^{\alpha \beta}+\frac{q_{i}^{2}}{2 m_{\alpha \beta}^{i}}-i d^{\alpha \beta} \omega_{\nu}\right) \Delta_{\alpha}^{*}(q) \Delta_{\beta}(q) .
$$

The frequency dependence has been kept, to lowest order in $\omega_{\nu}$, in order to look at the effects of quantum fluctuations.

The constant term is given by,

$$
r^{\alpha \beta}=\left(\delta^{\alpha \beta}-\frac{V}{2} \sum_{\epsilon} \frac{\tanh (\beta \xi / 2)}{\xi} N^{\alpha \beta}(\epsilon)\right),
$$

where the weighted densities of states (WDOS) are

$$
N^{\alpha \beta}(\epsilon)=\frac{1}{N} \sum_{\mathbf{k}} \zeta^{\alpha}(\mathbf{k}) \zeta^{\beta}(\mathbf{k}) \delta\left(\epsilon-\epsilon_{\mathbf{k}}\right) .
$$

For the nearest neighbour hopping square lattice the WDOS are plotted in Fig. A. As one can see from the figure the $d$-wave WDOS is strongly peaked around the normal state van Hove peak at half-filling, whilst the (extended) $s$-wave WDOS is suppressed at that point but large near the band edges.

Note that the square symmetry of the lattice forbids the existence of cross-terms such as $r^{s d}$ so that $N^{s d}$ is zero. Whilst $\zeta^{s}(\mathbf{k})$ is even under the exchange of $x$ - and $y$-momenta, $\zeta^{d}(\mathbf{k})$ is odd under this operation. The products $\zeta^{s} \zeta^{s}$ and $\zeta^{d} \zeta^{d}$ are both even but $\zeta^{s} \zeta^{d}$ is odd. Provided $\epsilon_{\mathbf{k}}$ is even under $x \leftrightarrow y, N^{s d}$ must be zero. However, if the $x \leftrightarrow y$ symmetry in $\epsilon_{\mathbf{k}}$ is broken, say by an orthorhombic distortion, then $r^{\text {sd }}$ need not be zero.

The masses come from the second derivatives (with respect to momentum) of the static susceptibilty, $\chi_{0}^{\alpha \beta}(\mathbf{q})=$ $\chi_{0}^{\alpha \beta}\left(i \omega_{\nu}=0, \mathbf{q}\right)$ at $\mathbf{q}=0$. Generally, for any given band structure, $\epsilon_{\mathbf{k}}$, they are,

$$
\begin{aligned}
\frac{1}{2 m_{\alpha \beta}^{i j}}=\frac{V}{8 N} \sum_{\mathbf{k}}\left[\frac { 1 } { m _ { i j } ^ { * } ( \mathbf { k } ) } \left(\frac{n_{f}^{\prime}\left(\xi_{\mathbf{k}}\right)}{\xi_{\mathbf{k}}}\right.\right. & \left.-\frac{n_{f}\left(\xi_{\mathbf{k}}\right)-\frac{1}{2}}{\xi_{\mathbf{k}}^{2}}\right) \zeta^{\alpha}(\mathbf{k}) \zeta^{\beta}(\mathbf{k}) \\
& \left.+v_{i}(\mathbf{k}) v_{j}(\mathbf{k})\left(\frac{n_{f}^{\prime \prime}\left(\xi_{\mathbf{k}}\right)}{\xi_{\mathbf{k}}}\right) \zeta^{\alpha}(\mathbf{k}) \zeta^{\beta}(\mathbf{k})\right],
\end{aligned}
$$

where a prime indicates differentiation and the band masses and quasiparticle velocities are,

$$
\frac{1}{m_{i j}^{*}(\mathbf{k})}=\left(\frac{\partial^{2} \epsilon_{\mathbf{k}}}{\partial k_{i} \partial k_{j}}\right), \quad v_{i}(\mathbf{k})=\left(\frac{\partial \epsilon_{\mathbf{k}}}{\partial k_{i}}\right)
$$

For the nearest neighbour square lattice only the diagonal terms, $m_{i i}^{*}(\mathbf{k})$ and $v_{i}^{2}(\mathbf{k})$, are non-zero and even:

$$
\frac{1}{m_{i i}^{*}(\mathbf{k})}=2 t \cos \left(k_{i}\right), \quad v_{i}(\mathbf{k})^{2}=4 t^{2} \sin ^{2}\left(k_{i}\right) .
$$

Unlike $r^{\alpha \beta}$, the mass in Eq. (23) is not purely diagonal in the flavour indices. In addition to the diagonal terms there are gradient mixing terms [29,30],

$$
\Delta_{s}^{*}(q)\left(\frac{q_{x}^{2}-q_{y}^{2}}{2 m_{s d}^{x}}\right) \Delta_{d}(q)+\Delta_{d}^{*}(q)\left(\frac{q_{x}^{2}-q_{y}^{2}}{2 m_{s d}^{x}}\right) \Delta_{s}(q),
$$

where $m_{s d}^{x}=-m_{s d}^{y}$. They are allowed because they have the symmetry of the square. Under an exchange $x \leftrightarrow y$ both the gradients and the $d$-wave order parameter change sign, leaving the whole expression unchanged.

For weak coupling, such terms have little effect except in the vicinity of inhomogeneities such as impurities or interfaces where the magnitude of the order parameter is expected to be diminished. In these regions, a gradient in the dominant order parameter would drive the subdominant order parameter to be non-zero. So, for instance, near the surface of a bulk $d$-wave superconductor an induced $s$-wave component might appear. When we consider the strong coupling limit we will see the importance of these gradient mixing terms even in the bulk. 
We now have expressions for $r^{\alpha \beta}$ and $m_{\alpha \beta}^{i}$, leaving only $d^{\alpha \beta}$ to be determined. The derivation of $d^{\alpha \beta}$ requires some care [23]. The Matsubara frequencies are discrete, therefore to expand in them we must first make the analytic continuation $\chi^{\alpha \beta}\left(i \omega_{\nu}, \mathbf{q}=0\right) \rightarrow \chi^{\alpha \beta}\left(\omega+i 0^{+}, \mathbf{q}=0\right)$ and then expand in $\omega$. We find,

$$
d^{\alpha \beta}=\frac{V}{4} \sum_{\epsilon} \frac{\tanh (\beta \xi / 2)}{\xi^{2}} N^{\alpha \beta}(\epsilon)-\frac{i \pi \beta V}{8} N^{\alpha \beta}(\mu) .
$$

Like $r^{\alpha \beta}$, the tensor $d^{\alpha \beta}$ is diagonal in the flavour indices. The real part of $d^{\alpha \beta}$ represents the free propagation of pairs, whilst the imaginary part represents the decay of pairs into the continuum of fermionic states. The significance of the two terms, and their domains of applicability will be discussed when we consider the effects of Gaussian fluctuations in section IV.

The quartic part of the action is shown in Fig. 3(ii). We associate a susceptibility with the fermion loop:

$$
\begin{aligned}
\chi_{4}^{\alpha \beta \gamma \delta} & =-\frac{1}{\beta N} \sum_{\mathbf{k}}\left|G_{0}\left(i \omega_{n}, \mathbf{k}\right)\right|^{4} \zeta^{\alpha}(\mathbf{k}) \zeta^{\beta}(\mathbf{k}) \zeta^{\gamma}(\mathbf{k}) \zeta^{\delta}(\mathbf{k}), \\
& =-\frac{1}{2 N} \sum_{\mathbf{k}}\left(\frac{n_{f}^{\prime}\left(\xi_{\mathbf{k}}\right)}{\xi_{\mathbf{k}}^{2}}-\frac{n_{f}\left(\xi_{\mathbf{k}}\right)-\frac{1}{2}}{\xi_{\mathbf{k}}^{3}}\right) \zeta^{\alpha}(\mathbf{k}) \zeta^{\beta}(\mathbf{k}) \zeta^{\gamma}(\mathbf{k}) \zeta^{\delta}(\mathbf{k}),
\end{aligned}
$$

where $\alpha, \beta, \gamma, \delta$ denote the flavour indices at each vertex. Only the diagrams with an even number of $s$ and $d$ indices contribute. Thus, including the rescaling of $\Delta$ by $V^{\frac{1}{2}}$, the bosonic action to fourth order reads,

$$
\begin{aligned}
\mathcal{S}^{(4)}=-\frac{1}{4} & \operatorname{Tr}\left(\mathbf{V G}_{0}\right)^{4}, \\
=-u_{s} & \sum_{\{q\}} \Delta_{s}^{*}\left(q_{1}\right) \Delta_{s}\left(q_{2}\right) \Delta_{s}^{*}\left(q_{3}\right) \Delta_{s}\left(q_{4}\right) \times \delta\left(q_{1}-q_{2}+q_{3}-q_{4}\right) \\
& -u_{d} \sum_{\{q\}} \Delta_{d}^{*}\left(q_{1}\right) \Delta_{d}\left(q_{2}\right) \Delta_{d}^{*}\left(q_{3}\right) \Delta_{d}\left(q_{4}\right) \times \delta\left(q_{1}-q_{2}+q_{3}-q_{4}\right) \\
& -2 u_{x} \sum_{\{q\}} \Delta_{s}^{*}\left(q_{1}\right) \Delta_{s}\left(q_{2}\right) \Delta_{d}^{*}\left(q_{3}\right) \Delta_{d}\left(q_{4}\right) \times \delta\left(q_{1}-q_{2}+q_{3}-q_{4}\right) \\
& -\frac{u_{x}}{2} \sum_{\{q\}} \Delta_{s}^{*}\left(q_{1}\right) \Delta_{d}\left(q_{2}\right) \Delta_{s}^{*}\left(q_{3}\right) \Delta_{d}\left(q_{4}\right) \times \delta\left(q_{1}-q_{2}+q_{3}-q_{4}\right) \\
& -\frac{u_{x}}{2} \sum_{\{q\}} \Delta_{d}^{*}\left(q_{1}\right) \Delta_{s}\left(q_{2}\right) \Delta_{d}^{*}\left(q_{3}\right) \Delta_{s}\left(q_{4}\right) \times \delta\left(q_{1}-q_{2}+q_{3}-q_{4}\right) .
\end{aligned}
$$

The three coefficients are,

$$
\begin{aligned}
& u_{s}=-\frac{V^{2}}{8 \beta N} \chi_{4}^{s s s s}, \\
& u_{d}=-\frac{V^{2}}{8 \beta N} \chi_{4}^{d d d d}, \\
& u_{x}=-\frac{V^{2}}{4 \beta N} \chi_{4}^{s s d d},
\end{aligned}
$$

where we note that $\chi_{4}^{s s d d}=\chi_{4}^{\text {sdsd }}$.

This completes our derivation of the Landau-Ginzburg-Wilson action. In the following sections we will examine the saddle point (mean field) and Gaussian fluctuation approximations to this action in order to derive the phase diagram of the model and to study the crossover to strong-coupling superconductivity.

\section{MEAN FIELD (LANDAU) THEORY}

In the limit that the superconducting fields are static and spatially uniform, the action, $\mathcal{S}_{b}$, is related to the Landau free energy for interacting $s$ - and $d$-wave superconductivity: 


$$
\begin{aligned}
\beta \mathcal{F}=-\mathcal{S}_{b}^{m f}=\frac{1}{2} r_{s}\left|\Delta_{s}\right|^{2}+\frac{1}{2} r_{d}\left|\Delta_{d}\right|^{2}+u_{s}\left|\Delta_{s}\right|^{4}+u_{d}\left|\Delta_{d}\right|^{4} & \\
& +2 u_{x}\left|\Delta_{s}\right|^{2}\left|\Delta_{d}\right|^{2}+\frac{u_{x}}{2}\left(\Delta_{s} \Delta_{s} \Delta_{d}^{*} \Delta_{d}^{*}+\text { c.c. }\right) .
\end{aligned}
$$

This form for the free energy is identical to that found by Feder and Kallin [29] and Joynt [30] who derived it from symmetry principles.

Rationalising the two cross-terms into one by writing the order parameters in terms of their phases and moduli, so that

$$
2 u_{x}\left|\Delta_{s}\right|^{2}\left|\Delta_{d}\right|^{2}+\frac{u_{x}}{2}\left(\Delta_{s} \Delta_{s} \Delta_{d}^{*} \Delta_{d}^{*}+\text { c.c. }\right)=2 u_{x}\left(1+\frac{1}{2} \cos (2 \Delta \theta)\right)\left|\Delta_{s}\right|^{2}\left|\Delta_{d}\right|^{2},
$$

we see that the free energy, $\mathcal{F}$, depends on the phase difference, $\Delta \theta$, between $\Delta_{s}$ and $\Delta_{d}$.

The saddle points of the free energy, $\mathcal{F}$, which for a uniform system correspond to Hartree-Gor'kov mean field theory, are found by simultaneously minimising $\mathcal{F}$ with respect to the magnitudes of the fields and the phase difference, i.e.,

$$
\frac{\partial \mathcal{F}}{\partial\left|\Delta_{s}\right|}=\frac{\partial \mathcal{F}}{\partial\left|\Delta_{d}\right|}=\frac{\partial \mathcal{F}}{\partial \Delta \theta}=0
$$

As a first approximation to solving these equations, we uncouple the two order parameters and, for given $V$, solve for $\mathrm{T}_{c}$ as a function of $\mu$ for each field independently of the other. In this approximation, Eq. (38) gives Stoner-like criteria for each of the critical temperatures:

$$
r_{\alpha}=0, \quad \alpha=s, d .
$$

It is not possible to find analytic solutions to these equations except in the large $V$ limit where we expect $\mathrm{T}_{c}$ to be large too. In this case we find,

$$
\lim _{V \rightarrow \infty} r_{\alpha}=1-\frac{V \beta}{4} \sum_{\epsilon} N^{\alpha \alpha}(\epsilon)
$$

which implies that, in this limit, the critical temperatures for both $s$ - and $d$-wave superconductivity are the same:

$$
\mathrm{T}_{c}=\frac{V}{4} .
$$

Computationally, however, the solution of $r_{\alpha}=0$ is a trivial matter for all $V$. The results are shown in Figs. D(a)-(c) for three different values. The maximum value of the ordinate has been chosen as $V / 4$ for each one so that the approach to the strong-coupling limit can be followed.

For small coupling, as in Fig. 5(a), the $s$-wave solutions have their highest $\mathrm{T}_{c}$ at the edges of the band whilst the $d$-wave solutions have theirs at the band centre. These places correspond to where the respective WDOS of each is the largest. The effect of the van Hove singularity in the centre of the $d$-wave WDOS is to produce a high transition temperature in that channel, just as would be predicted by conventional BCS theory in which it is the combination $V N^{\alpha \alpha}(0)$ that determines $\mathrm{T}_{c}$. In fact, it has been credibly suggested by some authors 31 33 that it is the van Hove singularity, observed in most first-principles calculations [34,35], combined with a conventional weakcoupling electron-phonon or spin-fluctuation mechanism, that is responsible for the high transition temperatures in the cuprate materials. For the extended $s$-wave channel there is no van Hove singularity and the transition temperatures are typically a factor of five lower than the $d$-wave ones. For small coupling, neither the $s$ - nor the $d$-wave critical temperatures are near the limiting value of $V / 4$

As $V$ is increased, Fig. 同(b), the $s$-wave solutions begin to occupy the centre of the band and the $d$-wave solutions spread outwards towards the band edges. The maximum $\mathrm{T}_{c}$ 's increasingly tend to the strong-coupling value, $V / 4$. As $V$ increases further towards the strong-coupling limit, Fig. 5 (c), the $s$ - and $d$-wave solutions start to converge. This is because the exact shape of the WDOS becomes less important as more of it falls within $k_{B} \mathrm{~T}_{c}$ of the Fermi surface. Note that for large $V$ both the $s$ - and $d$-wave solutions have spread beyond the edges of the band. This, and the crossover to local pairs, will be discussed when we consider the effects of Gaussian fluctuations in the next section.

Now that the phase diagram of the uncoupled order parameters is known, we turn our attention to the coupled case. From Figs. 5(b) and (c) we see that there are regions where both the uncoupled $s$ - and $d$-wave order parameters are simultaneously below their critical temperatures. What is the nature of the pairing in these regions? According to the values of the model parameters, two cases may be distinguished: (i) For $u_{s} u_{d}<u_{x}^{2}\left(1+\frac{1}{2} \cos (2 \Delta \theta)\right)^{2}$ the 
pure $s$ - and $d$-wave phases are separated by a single first order transition which meets the two second order metalsuperconductor lines at a bicritical point; (ii) For $u_{s} u_{d}>u_{x}^{2}\left(1+\frac{1}{2} \cos (2 \Delta \theta)\right)^{2}$ an intermediate phase of mixed symmetry $\left(\Delta_{s} \neq 0, \Delta_{d} \neq 0\right)$ is permissible, separated from each of the two pure phases by a line of continuous second order transitions. These meet the metal-superconductor lines at a tetracritical point.

In the mixed phase the value of the phase difference, $\Delta \theta$, is of paramount importance. It is easily found from Eq. (38). Depending on the sign of $u_{x}$ it can assume either of two values:

$$
\Delta \theta=\left\{\begin{array}{cl}
\pi / 2 & u_{x}>0 \Rightarrow s \pm i d \\
0 & u_{x}<0 \Rightarrow s \pm d
\end{array}\right.
$$

The two solutions represent fundamentally different phases. For instance, the $s \pm d$ phase is invariant under time reversal, whilst the $s \pm i d$ phase breaks this symmetry (time reversal involves complex conjugation). Several authors have suggested that the existence of a time reversal symmetry broken order parameter might be associated with spontaneous generation of currents at twin boundaries [36] or surfaces [37]. This effect has been observed experimentally as a splitting of the zero bias conductance peak in zero magnetic field [38]. No such effect would be observed in the $s \pm d$ phase.

Assuming that we are below the critical temperatures of both the $s$ - and $d$-wave order parameters, all possible states of the model can be parametrised by just two dimensionless variables,

$$
\begin{aligned}
U_{s} & =\frac{u_{s}}{u_{d}}\left|\frac{r_{d}}{r_{s}}\right|^{2}, \\
U_{x} & =\frac{u_{x}\left(1+\frac{1}{2} \cos (2 \Delta \theta)\right)}{u_{d}}\left|\frac{r_{d}}{r_{s}}\right|,
\end{aligned}
$$

where we remember that $\Delta \theta$ depends on the sign of $U_{x}$.

As the values of these two parameters vary there are transitions between the pure $s$ - and $d$-wave states and the two different mixed phases (separated by a line of first order transitions at $U_{x}=0$ ). The stable superconducting phases are shown in Fig. 6 as a function of $U_{s}$ and $U_{x}$.

For $V=5 t$, the variables $\left(U_{s}, U_{x}\right)$ were evaluated along a path in $(\mu, \mathrm{T})$ space shown in Fig. 5(b) as a dotted line. The resulting values are plotted as the dashed trajectory in 6. Starting in the pure $d$-wave sector at $\mathrm{T}=\mathrm{T}_{c}^{s}$, we remain in the pure $d$ state even though we are below $\mathrm{T}_{c}^{s}$ : the $s$-wave is suppressed by the dominant $d$-wave order parameter. Eventually we cross the second order phase boundary $(d \rightarrow s \pm i d)$ into the time reversal symmetry breaking mixed $s+i d$ state. With increasing $\mu$ the proportion of $s$-wave in the admixture rises and the $d$-wave portion is diminished until the, now dominant, $s$-wave totally supresses the $d$-wave and the second transition $(s \pm i d \rightarrow s)$ occurs. The trajectory terminates in the $s$-wave sector when $\mathrm{T}=\mathrm{T}_{c}^{d}$. We observe qualitatively identical behaviour for all $V$.

The other, $s+d$, mixed phase does not occur spontaneously for any parameters derived from the Hubbard model because of the form of the quartic susceptibility, Eq. (31), which ensures that $u_{x}$ is always positive. To see an $s+d$ phase it is necessary to include an orthorhombic distortion in the lattice, so that the hoppings in the $x$ and $y$ directions are different. Orthorhombic anisotropy, as present in YBCO, introduces an extra term into the free energy proportional to $\Delta_{s}^{*} \Delta_{d}+\Delta_{d}^{*} \Delta_{s}$. With a term like this a finite value for one of the components drives the other to be non-zero too. This effect has been studied elsewhere [36] so we do not consider it here.

\section{GAUSSIAN FLUCTUATION THEORY}

It is clear from Figs. 5 (a)-(c) that, as the coupling $V$ increases, $\mathrm{T}_{c}$ starts to become finite even below the bottom (top) of the electronic band at $\mu=-4 t(+4 t)$. For such values of the chemical potential, fermions can only exist as bound pairs, not as single particles. A similar phenomenon has been noted for the local $s$-wave case in Jellium by several authors 20 22,39] who concluded that, in the low density limit, Bose-Einstein condensation of these pairs constitutes the mechanism for superconductivity.

As $V$ increases, the mean field approximation becomes increasingly poor and the effects of fluctuations must be included. Specifically we want to know how $\mathrm{T}_{c}$, as a function of filling, is affected. As it stands, the functional, $\mathcal{S}_{b}$, is too complicated. We therefore employ the Gaussian approximation in which terms of $\mathcal{O}\left(|\Delta|^{4}\right)$ and above are neglected. The free energy can be evaluated exactly in this approximation. The action is,

$$
\mathcal{S}_{b}=-\frac{1}{2} \sum_{q}\left(r^{\alpha \beta}+\frac{q_{i}^{2}}{2 m_{\alpha \beta}^{i}}-i d^{\alpha \beta} \omega_{\nu}\right) \Delta_{\alpha}^{*}(q) \Delta_{\beta}(q)
$$


There are two important limits to consider: weak coupling/high density $(|\mu|<4 t)$ and strong coupling/low density $(|\mu|>4 t)$. Weak coupling corresponds to BCS-like Cooper-pair superconductivity. In this limit the imaginary part of $d$ is dominant so the dynamics are relaxational: pairs have a finite lifetime after which they decay into the continuum of fermionic states. As the coupling strength is increased, or the density is lowered, the chemical potential can fall below the bottom of the band. In this case the imaginary part of $d$ is zero, see Fig. if, so the pairs become infinitely long lived and the physics is that of propagating pairs. This is the Bose limit. The manner in which the crossover comes about is quite different for the $s$ - and $d$-wave superconductors because of form of the WDOS is different for each. The $s$-wave WDOS (see Fig. (1) shows a sharp band-edge so that the transition from just inside to just outside the band is sharp too, whilst the $d$-wave WDOS (see Fig. (1) goes smoothly to zero so the transition is smooth.

Although our theory provides an interpolation scheme between the strongly and weakly interacting regimes, its validity in the crossover region, near $|\mu|=4$, is doubtful. In this regime, fluctuation corrections [40] of order $k_{F} a_{F}$ (where $k_{F}$ is the Fermi vector and $a_{F}$ is the fermion scattering length), which are vanishingly small in the extreme limits, become large signaling a breakdown of the theory. In the preceeding sections we studied the weak limit in some detail. In this section we will go on to examine the strong coupling limit, omitting the intermediate regime. This is regrettable, especially as it is just this regime that is most relevant to the cuprates, but unavoidable within the current formulation.

\section{A. On-site (local) pairing}

As a test of the performance of the Gaussian theory we will first consider the on-site, local $s$-wave superconducting Hubbard model. The Gaussian action for it is,

$$
\mathcal{S}_{b}=-\frac{1}{2} \sum_{q}\left(r+\frac{\mathbf{q} \cdot \mathbf{q}}{2 m}-i d \omega_{\nu}\right)\left|\Delta_{q}\right|^{2},
$$

where $\Delta_{q}$ is the local $s$-wave field and the parameters, $r, m$ and $d$, are found from those of the nearest neighbour extended Hubbard model by replacing $V$ with $U$ and setting $\zeta^{\text {local } s}(\mathbf{k})=1$. Scaling the fields, we can set the frequency coefficient to unity, in which case the action resembles that of free bosons:

$$
\mathcal{S}_{b}=-\frac{1}{2} \sum_{q}\left(-\mu_{b}+\frac{\mathbf{q} \cdot \mathbf{q}}{2 M}-i \omega_{\nu}\right)\left|\Delta_{q}\right|^{2},
$$

with $\mu_{b}=-r / d$ the bosonic chemical potential, the zero of which signals the onset of Bose-Einstein condensation, and $M=m d$ the boson mass. It is well known [41] that as the on-site coupling, $U$, increases, so too does the mass of the bosons. This can be understood by considering the mechanism of the bosonic hopping. The hopping of a pair consists of three stages: (i) A pair occupies a single site. (ii) Virtual ionisation - one electron hops onto a neighbouring site, gaining a kinetic energy, $t$, but sacrificing the pair-binding energy, $U$. (iii) Pair recombination - the second electron hops onto the neighbouring site too, gaining a kinetic energy, $t$, and pair-binding energy, $U$. The matrix element for the whole hopping process, proportional to one over the boson mass, is $t^{2} / U$. We now show that our Gaussian theory can produce this effect.

In the extreme large $U$ limit, where the density is very low and $U / \mathrm{T}_{c} \gg 1$, the electronic chemical potential equals half the binding energy of a Bose pair:

$$
\lim _{U \rightarrow \infty} \mu=-\frac{U}{2} .
$$

In the same limit we find the frequency coefficient,

$$
\lim _{U \rightarrow \infty} d=\frac{U}{4} \sum_{\epsilon} \frac{1}{\xi^{2}} N(\epsilon)=\frac{U}{4 \mu^{2}}=\frac{1}{U},
$$

and the unscaled mass,

$$
\begin{aligned}
\lim _{U \rightarrow \infty} \frac{1}{2 m^{i}} & =\frac{U}{16 N} \sum_{\mathbf{k}} \frac{1}{m_{i i}^{*}(\mathbf{k})} \frac{1}{\xi_{\mathbf{k}}^{2}} \\
& =\frac{U}{16 N} \sum_{\mathbf{k}} \frac{1}{m_{i i}^{*}(\mathbf{k})}\left(\frac{1}{\mu^{2}}+\frac{2 \epsilon_{\mathbf{k}}}{\mu^{3}}+\ldots\right) \\
& =\frac{2 t^{2}}{U^{2}}
\end{aligned}
$$


where the first term in the expansion of $1 / \xi_{\mathbf{k}}^{2}$ integrated to zero. Putting these together gives the asymptotic behaviour of the boson mass:

$$
M=\frac{U}{4 t^{2}},
$$

which is exactly as predicted by the virtual ionisation arguments.

We now evaluate the free energy. With the action in Gaussian form the functional integral can be performed exactly to give,

$$
\begin{aligned}
\beta \mathcal{F}_{b}=-\ln \int \mathcal{D}\left[\Delta^{*}, \Delta\right] e^{\mathcal{S}_{b}} & =-\ln \operatorname{Det}^{-1}\left(-\mu_{b}+\frac{\mathbf{q} \cdot \mathbf{q}}{2 M}-i \omega_{\nu}\right), \\
& =\operatorname{Tr} \ln \left(-\mu_{b}+\frac{\mathbf{q} \cdot \mathbf{q}}{2 M}-i \omega_{\nu}\right) .
\end{aligned}
$$

For given $\mu$ and $U$ Gaussian fluctuations do not alter $\mathrm{T}_{c}$, but this is not to say that they do not have any effect at all. Following Randeria et al. [39], who examined an $s$-wave continuum model, we calculate the fluctuation contribution to the fermion number density, $n\left(\mu, T_{c}\right)$. For fixed $n$, the changes in $\mu\left(n, \mathrm{~T}_{c}\right)$ effectively renormalises $\mathrm{T}_{c}(n)$.

The fermion number density, $n$, is derived from the free energy, $\mathcal{F}=\mathcal{F}_{0}+\mathcal{F}_{b}$, by differentiation with respect to the chemical potential, $\mu$ :

$$
n=n_{0}+\delta n=-\frac{\partial \mathcal{F}}{\partial \mu} .
$$

The contribution from the free fermion action, $\mathcal{F}_{0}$, is

$$
n_{0}=1-\sum_{\epsilon} \tanh (\beta \xi / 2) N(\epsilon)
$$

In the BCS regime, $|\mu|<4, \delta n$ is negligible and the mean field results are unaltered. As we approach the band edge, $|\mu|=4$, where our theory is not strictly valid, the fluctuation contribution starts to become important. In the Bose limit, $|\mu|>4$, the fluctuation contribution to $n$ is considerable. In fact, in the $U \rightarrow \infty$ limit $n\left(\mu, \mathrm{T}_{c}\right)=\delta n\left(\mu, \mathrm{T}_{c}\right)$. The boson contribution is,

$$
\begin{aligned}
\delta n & =\frac{1}{\beta} \frac{\partial \mu_{b}}{\partial \mu} \sum_{\mathbf{q}} \sum_{\nu} \frac{1}{-\mu_{b}+\mathbf{q} \cdot \mathbf{q} / 2 M-i \omega_{\nu}}, \\
& =\frac{\partial \mu_{b}}{\partial \mu} \sum_{\mathbf{q}} n_{b}\left(-\mu_{b}+\mathbf{q} \cdot \mathbf{q} / 2 M\right),
\end{aligned}
$$

where the Bose occupation number is $n_{b}(x) \equiv\left[e^{\beta x}-1\right]^{-1}$. For simplicity we have ignored small contributions from terms proportional to the derivatives of $d$ and $m$ which tend to zero as $U$ increases.

The partial derivative of $r$ is,

$$
\frac{\partial r}{\partial \mu}=-\frac{U}{2} \sum_{\epsilon}\left(\frac{\tanh (\beta \xi / 2)}{\xi^{2}}+\frac{2 n^{\prime}(\xi)}{\xi}\right) N(\epsilon)
$$

which, for $\mathrm{T}=\mathrm{T}_{c}$, leads to the useful result that,

$$
\lim _{U \rightarrow \infty} \frac{\partial \mu_{b}}{\partial \mu}=2 .
$$

Combining this result with Eq. (59) tells us that, in the low density, strong interaction limit, the fermion density at the critical temperature is,

$$
n\left(\mu, \mathrm{T}_{c}\right)=2 \sum_{\mathbf{q}} n_{b}(\mathbf{q} \cdot \mathbf{q} / 2 M),
$$

i.e., all the fermions are bound into Bose pairs of mass $M$.

Unfortunately, there is a fly in the ointment: in two dimensions the integral in Eq. (62) has an infrared logarithmic divergence due to soft $\mathbf{q}=0$ modes. This problem can be circumvented be remembering that the cuprate materials 
are not really two dimensional; rather they are highly anisotropic 3D materials. To take account of this we make the substitution

$$
\frac{\mathbf{q} \cdot \mathbf{q}}{2 M} \equiv \frac{q_{x}^{2}}{2 M}+\frac{q_{y}^{2}}{2 M} \longrightarrow \frac{q_{x}^{2}}{2 M}+\frac{q_{y}^{2}}{2 M}+a^{2} \frac{q_{z}^{2}}{2 M},
$$

where $a^{2} \equiv M / M^{z} \ll 1$, reflecting the low mobility of electrons between $\mathrm{CuO}_{2}$ planes. With this substitution $n$ is always finite. Assuming $\mathrm{T}_{c}<\frac{a^{2} \pi^{2}}{2 M}$ we can safely take the limits of the $\mathbf{q}$ integral to infinity, giving,

$$
n=\frac{\zeta(3 / 2)}{a}\left(\frac{M \mathrm{~T}_{c}}{\pi \sqrt[3]{2}}\right)^{\frac{3}{2}}
$$

where $\zeta(\cdot)$ is the Riemann Zeta function. Aside from the $1 / a$ prefactor which accounts for the mass anisotropy, this is exactly the result we would expect for a $3 \mathrm{D}$ gas of mass $M$ bosons with Bose-Einstein condensation temperature $\mathrm{T}_{c}$. Rearranging this equation gives an expression for $\mathrm{T}_{c}$ at fixed density:

$$
\mathrm{T}_{c}=2.09 \frac{(a n)^{2 / 3}}{M} .
$$

From this equation we can see the importance of the mass: a large mass means a low $\mathrm{T}_{c}$ and vice versa. Thus, for the large $U$ limit in which we are interested, increasing the coupling, which would "normally" (i.e., in mean field talk) increase the critical temperature, has the paradoxical effect of lowering $\mathrm{T}_{c}$ due to the localising effect it has on the pairs.

We expect to see analogous behaviour in our extended Hubbard model as we increase the coupling, but instead of becoming localised on a site, our bosons should become localised on bonds, becoming more and more massive, i.e., less mobile, as $V$ increases.

\section{B. Nearest neighbour (non-local) pairing}

The analysis for the extended Hubbard model proceeds along similar lines as the on-site model but is complicated by the existence of two species of bosons, $\Delta_{s}$ and $\Delta_{d}$, or, equivalently, $\Delta_{x}$ and $\Delta_{y}$. In the large $V$ limit we find,

$$
\begin{aligned}
\lim _{V \rightarrow \infty} d^{\alpha \beta} & =\frac{\delta_{\alpha \alpha}}{V} \\
\lim _{V \rightarrow \infty} \frac{1}{2 m_{\alpha \beta}^{i}} & =\frac{V}{16 N} \sum_{\mathbf{k}} \frac{\zeta^{\alpha}(\mathbf{k}) \zeta^{\beta}(\mathbf{k})}{m_{i i}^{*}(\mathbf{k})} \frac{1}{\xi_{\mathbf{k}}^{2}} \\
& =\frac{V}{16 N} \sum_{\mathbf{k}} \frac{\zeta^{\alpha}(\mathbf{k}) \zeta^{\beta}(\mathbf{k})}{m_{i i}^{*}(\mathbf{k})}\left(\frac{1}{\mu^{2}}+\frac{2 \epsilon_{\mathbf{k}}}{\mu^{3}}+\ldots\right) .
\end{aligned}
$$

For nearest neighbour hopping, these give the following bosonic masses:

$$
\left(M_{\alpha \beta}^{x}, M_{\alpha \beta}^{y}\right)=\frac{V}{t^{2}} \begin{cases}\left(\frac{1}{9}, \frac{1}{9}\right) & \alpha \beta=s s \\ (1,1) & \alpha \beta=d d \\ (1,-1) & \alpha \beta=s d .\end{cases}
$$

The anisotropy between the $s$ and $d$ boson masses arises because the form factors for the $s$ - and $d$-wave bosons, $\zeta^{s} \zeta^{s}$ and $\zeta^{d} \zeta^{d}$, differ by the sign on $\cos \left(k_{x}\right) \cos \left(k_{y}\right)$. (This term does not enter the $s d$ cross term.) This is best understood in terms of the $x$ and $y$ fields. In that basis we see that the term comes from the process, shown in Fig. 8(i), by which an $x$ field becomes a $y$ field. Obviously such a process is sensitive to the relative phases of the two bonds: zero phase difference is optimal for the hopping to occur so the $s$-wave phase is preferred.

At this point, a pertinent question is, what happens if we change the bandstructure? As an example, consider adding a next nearest neighbour hopping, $t^{\prime}$, to the Hamiltonian so that the band energy is,

$$
\epsilon_{\mathbf{k}}=-2 t\left(\cos \left(k_{x}\right)+\cos \left(k_{y}\right)\right)-4 t^{\prime} \cos \left(k_{x}\right) \cos \left(k_{y}\right)
$$

The diagonal hopping allows the $x \leftrightarrow y$ process to occur without a potential energy cost: there is no intermediate state where $V$ is lost. See Fig. $8(i i)$ 
In terms of the mathematics, the $t^{\prime}$ hopping means that the integrand for the $1 / \mu^{2}$ term in the expansion of $1 / \xi_{\mathbf{k}}^{2}$ goes like $\pm t^{\prime} \cos ^{2}\left(k_{x}\right) \cos ^{2}\left(k_{y}\right)$ which has the same sign over the whole range of integration and is therefore finite (c.f. the nearest neighbour hopping contribution at this order which has only odd powers of $\cos \left(k_{x}\right)$ and $\cos \left(k_{y}\right)$ and therefore integrates to zero). The masses calculated with this term are:

$$
\frac{1}{M_{\alpha \alpha}\left(t, t^{\prime}\right)}= \begin{cases}\frac{1}{V}\left(9 t^{2}+t^{\prime} V\right) & \alpha=s \\ \frac{1}{V}\left(t^{2}-t^{\prime} V\right) & \alpha=d\end{cases}
$$

If the product $t^{\prime} V$ is much smaller than $t^{2}$ the nearest neighbour hopping can be safely ignored. However, if this is not the case then the corrections can be of great significance. For instance, if $t^{\prime} V \simeq t^{2}$ then the $d$-wave solution is unstable (has negative mass), or if $t^{\prime} V \simeq-9 t^{2}$ then the $s$-wave solution is unstable. In the limit $\left|t^{\prime} V\right| \gg t^{2}$ only one flavour of boson is stable and its mass is independent of $V$ :

$$
\lim _{\left|t^{\prime} V\right| \rightarrow \infty} M\left(t, t^{\prime}\right)=\frac{1}{t^{\prime}}
$$

Including a sufficiently large diagonal hopping, $t^{\prime}$, obviates the need for pair hopping to occur via virtual ionisation. Once this requirement has been lifted, the lattice model behaves exactly as we would expect a continuum model to behave (as shown in Fig. 1).

Assuming for the moment that both boson modes are stable (i.e., $t^{\prime}=0$ or small) we resume our calculation. The Gaussian free energy is,

$$
\begin{aligned}
\beta \mathcal{F}_{b} & =\operatorname{Tr} \ln \left(-\mu_{b}^{\alpha \beta}+\frac{q_{i}^{2}}{2 M_{\alpha \beta}^{i}}-i \omega_{\nu}\right), \\
& =\sum_{q} \ln \left(\lambda_{+} \lambda_{-}\right)
\end{aligned}
$$

where we have used the cyclic nature of the trace to diagonalise the action with respect to its flavour indices. The eigenvalues, $\lambda_{ \pm}$, are the energies of the eigenmodes of the system which in general are linear combinations of $s$ and $d$ (or $x$ and $y$ ) modes. In the large $V$ limit, the eigenvalues are,

$$
\lambda_{ \pm}=-\frac{1}{2}\left(\mu_{s s}^{*}+\mu_{d d}^{*}\right)+\frac{|\mathbf{q}|^{2}}{2 M_{ \pm}(\theta)}-i \omega_{\nu}
$$

where the masses, which depend on the angle $\theta=\tan ^{-1}\left(\frac{q_{y}}{q_{x}}\right)$, are,

$$
\frac{1}{M_{ \pm}(\theta)}=\frac{1}{M}\left(5 \pm \sqrt{17 \cos ^{4}(\theta)+30 \cos ^{2}(\theta) \sin ^{2}(\theta)+17 \sin ^{4}(\theta)}\right)
$$

with $M=M_{d d}=V / t^{2}$ as a convenient mass unit.

With these definitions the bosonic number density is,

$$
n\left(\mu, \mathrm{T}_{c}\right)=\frac{1}{2 \pi} \int_{-\pi}^{+\pi} d \theta\left\{n_{+}(\theta)+n_{-}(\theta)\right\},
$$

where the densities of the two modes, as functions of angle in $\mathbf{q}$-space, are

$$
n_{ \pm}(\theta)=\sqrt{\frac{2}{\pi}} \frac{\zeta(3 / 2)}{a}\left(M_{ \pm}(\theta) \mathrm{T}_{c}\right)^{\frac{3}{2}}
$$

We see from this that for given $n$ and $\mathrm{T}_{c}$, the mode with the largest mass will be the most populous. This is graphically illustrated in Fig. 9 which shows the relative contributions of the two masses to the angle-dependent density.

The flavour of boson with the smaller of the two masses, $M_{+}(\theta)$, contributes negligibly $(\simeq 3 \%)$ to the total number density, therefore the physics is determined by the boson with the largest mass, $M_{-}(\theta)$. From Fig. 9 we see that the $M_{-}(\theta)$ bosons are not distributed evenly throughout space - they prefer to lie along one or other of the coordinate axes. Relative to an isotropic state, the bosons have been shifted away from the diagonals and onto the axes. For $M_{-}(\theta)$ as derived here, the effect is not very large $(\simeq 10 \%$ of the total), but by adjusting slightly the masses, for instance by setting $M_{s s} \simeq M_{d d}$, nearly all the weight can be shifted to the axes, leading to a very pronounced anisotropy. 


\section{DISCUSSION}

To understand how this might relate to the cuprates it is helpful to take a step back. In the derivations above we have assumed that all the electrons are bound into Bose pairs, which is reasonable provided $\mathrm{T}_{c} / V \ll 1$. However, for higher temperatures, where $\mathrm{T} / V \simeq 1$, this assumption is not valid as thermal excitations lead to the break-up of pairs. In the extreme high temperature regime almost all the electrons are free. Now, starting in the high temperature regime, as the temperature is lowered, the pairing fluctuations become more important and bosons start to form. In the Gaussian approximation, the onset of fluctuations is gradual, with a maximum at $\mathrm{T}_{c}$. $\mathrm{T}^{*}$ can be interpreted as the temperature where the fluctuations become significant, which is not necessarily the same as $\mathrm{T}_{c}^{M F}$. As the Bose number, $\delta n$, increases, the fermionic spectral weight is correspondingly decreased according to Eq. (56). Given the strongly anisotropic Bose number in Fig. 9 we can expect a similar anisotropy in the pseudogap. Interestingly, the Bose number is maximum along the axes and minimum on the diagonals which is just the same as the angle dependence of the pseudogap observed by ARPES measurements. Lowering the temperature further leads to the creation of more bosons and the pseudogap becomes deeper. Finally, at the Bose-Einstein condensation temperature a real $d$-wave superconducting gap will start to open, the maxima of which "accidentally" coincide with the pseudogap regions where there are most bosons.

It is important here to make two comments. Firstly, the pseudogap which we observe is not a superconducting effect in the sense that its origin does not lie in the spontaneous symmetry breaking of the superconducting order parameter, but rather in the formation of two-particle states which "eat" the available single-particle spectral weight in certain directions. Secondly, although the angular variation of the pseudogap is intimately related to the presence of $d$-wave pairs, the relation between the two is not as simple as might first appear. A single flavour order parameter, either $s$-wave or $d$-wave, must have an isotropic mass tensor (for $d$-wave this is shown as the dotted circle in Fig. 9), otherwise the theory will not be invariant under the square symmetry of the lattice. The angular anisotropy of the pseudogap comes not from the symmetry of a single order parameter, but from a coupling of the $d$-wave to the $s$-wave bosons via the anisotropic gradient, $\Delta_{d}^{*}\left(\nabla_{x}^{2}-\nabla_{y}^{2}\right) \Delta_{s}+c . c$. , which has the full square symmetry of the lattice. Equivalently we could say that the angular anisotropy is a reflection of the anisotropy in the masses of the $x$ and $y$ bosons: an $x$ boson prefers to travel in the $x$ direction and a $y$ boson prefers to travel in the $y$ direction. Were the $x$ boson to be totally confined to the $x$-axis, and the $y$ boson to the $y$-axis, in which case $M_{s s}=M_{d d}=M$, the angular distribution of the bosons would be sharply peaked along the axes and zero on the diagonals.

We have seen that it is necessary to have both $s$ - and $d$-wave order parameters if the pseudogap is to have any angular dependence, so it is natural to enquire about the nature of the superconducting state formed below $\mathrm{T}_{c}$. In the preceeding calculations it has been assumed that in the low density, large coupling limit, the critical temperatures for the $s$ - and $d$-wave fields become degenerate. Now, just because two order parameters have the same $\mathrm{T}_{c}$ does not mean that they will be present in the condensate in equal measure. Ignoring the gradient coupling for the moment, by analogy with Eq. (77) it is easy to see that, in a mixture of the two pure states, the heavier $d$ bosons will dominate by a ratio of $M_{s}^{3 / 2}: M_{d}^{3 / 2}=1: 27$. Including the gradient coupling terms the modes are admixtures of $s$ and $d$ bosons. The relative components can be found from the eigenvectors of the flavour matrix:

$$
\mathbf{e}^{ \pm}(\theta)=\left(\begin{array}{c}
\mathrm{e}_{s}^{ \pm}(\theta) \\
1
\end{array}\right)
$$

where the $s$ component is,

$$
\mathrm{e}_{s}^{ \pm}(\theta)=\frac{4 \pm \sqrt{17 \cos ^{4}(\theta)+30 \cos ^{2}(\theta) \sin ^{2}(\theta)+17 \sin ^{4}(\theta)}}{\cos ^{2}(\theta)-\sin ^{2}(\theta)} .
$$

For the dominant mode, Fig. 10 shows the variation of the $s$ component as a function of angle. (For the subdominant mode the same graph is produced by plotting $1 / \mathrm{e}_{s}^{+}(\theta)$.) For momenta lying along, or near, the diagonals, the dominant mode has an entirely $d$-wave character. As we move away from the diagonals and towards the $x$ and $y$ axes, the $s$-wave fraction grows, but even at its maximum value it is an order of magnitude smaller than the $d$-wave. We conclude that the dominant mode is essentially $d$-wave, whilst the subdominant one is essentially $s$-wave.

Thus, by including both the $s$ - and $d$-wave order parameters in the same theory, as well as the gradient couplings between them, we have been able to produce a pseudogap state with a non-trivial angular dependence, qualitatively similar to that of the $d$-wave superconducting state. Although the angle dependent pseudogap state requires an $s-d$ admixture, the superconducting state that it eventually gives way to has, for most intents and purposes, pure $d$-wave symmetry. 


\section{CONCLUSION}

In this paper we have considered an extended Hubbard model with nearest neighbour attractive interaction as a phenomenological model for the high $\mathrm{T}_{c}$ superconducting cuprates. By introducing a tensorial version of the Hubbard-Stratonovič transformation we were able to derive an effective Landau-Ginzburg-Wilson action describing both extended $s$ - and $d$-wave superconductivity.

The saddle point (mean field) approximation to the full action gave the phase diagram of the model as a function of chemical potential (filling) and temperature. Near the centre of the band $d$-wave solutions were found to be dominant whilst the extended $s$-wave solutions were dominant near the band edges. The two flavours of superconductivity were separated from each other by two second order phase transitions, in between which there was found to exist a mixed time-reversal symmetry breaking $s \pm i d$ phase. The alternative $s \pm d$ phase was ruled out except in the presence of orthorhombic distortions. At large values of the coupling, non-zero critical temperatures were observed beyond the band edges, signaling the creation of bosonic bound states.

Using the Gaussian approximation to the LGW action, in the low density limit, where quartic terms representing interactions between bosons ought to be unimportant, we first reproduced the known large $U$ behaviour of the on-site attractive Hubbard model, in which the bosons acquire a mass proportional to the coupling, $U$, due to the process of virtual ionisation. In the equivalent, large $V$, limit the extended model showed qualitatively similar behaviour provided only nearest neighbour hopping was considered. Introducing a next nearest neighbour hopping, $t^{\prime}$, allowed diagonal hopping processes, circumventing the need for virtual ionisation. It was shown that, in the limit $\left|t^{\prime} V\right| \gg t^{2}$, there is only one flavour of stable boson, $s$-wave if $t^{\prime}>0$ or $d$-wave if $t^{\prime}<0$, and that its mass is independent of $V$, just like in a continuum model. It was then argued that, for $\left|t^{\prime} V\right| \ll t^{2}$, the existence of two different bosonic modes, with anisotopic gradient couplings, could lead to an angle dependent distribution of bosons. This was then given a possible interpretation as the cause of the pseudogap state: two-particle states "eat" the single-particle spectral weight in certain areas of momentum space. Finally it was shown that, despite the mixing of the two different boson flavours, the superconducting ground state would be of predominantly $d$-wave character.

\section{ACKNOWLEDGEMENTS}

We would like to thank B.L. Györffy, J.J. Hogan-O'Neill and A.M. Martin for useful discussions. This work was partly supported by EPSRC under grant number GR/L22454.

[1] J.F. Annett, N.D. Goldenfeld, and A.J. Leggett, in Physical Properties of High Temperature Superconductors V (World Scientific, Singapore, 1996).

[2] A.G. Loeser et al., Science 273, 325 (1996).

[3] H. Ding et al., Nature 382, 51 (1996).

[4] W.N. Hardy et al., Phys. Rev. Lett. 70, 3999 (1993).

[5] C. Panagopolous et al., Phys. Rev. B 53, R2999 (1996).

[6] S.F. Lee et al., Phys. Rev. Lett. 77, 735 (1996).

[7] D.A. Wollman et al., Phys. Rev. Lett. 71, 2134 (1993).

[8] C.C. Tsuei et al., Phys. Rev. Lett. 73, 593 (1994).

[9] R.J. Kelley et al., Science 271, 1255 (1996).

[10] R.A. Klemm, M. Ledvij, and L. Shu., Chinese J. Phys. 34, 201 (1996).

[11] P. Monthoux and D. Pines., Phys. Rev. Lett. 67, 3448 (1991).

[12] G. Varelogiannis., Phys. Rev. B 57, R732 (1998).

[13] S.M. Anlage et al., Phys. Rev. B 50, 523 (1994).

[14] B. Batlogg et al., Physica C 235, 130 (1994).

[15] W.W. Warren (Jr.) et al., Phys. Rev. Lett. 62, 1193 (1989).

[16] M. Takigawa et al., Phys. Rev. B 43, 247 (1991).

[17] C.C. Homes et al., Phys. Rev. Lett. 71, 1645 (1993).

[18] A.V. Puchkov et al., Phys. Rev. Lett. 77, 3212 (1996).

[19] V.J. Emery and S.A. Kivelson, Nature 374, 434 (1995).

[20] P. Nozières and S. Schmitt-Rink, J. Low Temp. Phys. 59, 195 (1985). 
[21] M. Dreschler and W. Zwerger, Ann. Physik 1, 15 (1992).

[22] C. Sá de Melo, M. Randeria, and J.R. Engelbrecht, Phys. Rev. Lett. 71, 3202 (1993).

[23] M. Randeria, in Bose-Einstein Condensation (Cambridge University Press, Cambridge, 1995), Chap. 15.

[24] J. Hubbard, Phys. Rev. Lett. 3, 416 (1959).

[25] R.L. Stratonovič, Dokl. Akad. Nauk. SSSR 115, 1907 (1957).

[26] G.E. Volovik and L.P. Gor'kov, Sov. Phys. JETP 61, 843 (1985).

[27] G.M. Luke et al., Nature 394, 558 (1998).

[28] J.F. Annett, Adv. Phys. 39, 83 (1990).

[29] D.L. Feder and C. Kallin, Phys. Rev. B 55, 559 (1997).

[30] R. Joynt, Phys. Rev. B 41, 4271 (1990).

[31] J. Friedel, J. Phys.: Condens. Matter 1, 7757 (1989).

[32] D.M. Newns, C.C. Tsuei, and P.C. Pattnaik, Phys. Rev. B 52, 13611 (1995).

[33] G. Litak et al., Physica C 309, 257 (1998).

[34] O.K. Andersen, O. Jepsen, and A.I. Liechtenstein, Phys. Rev. B 49, 4145 (1994).

[35] D.L. Novikov and A.J. Freeman, in Recent Developments in High Temperature Superconductivity, edited by J. Klamut, B.W. Veal, B.M. Dabrowski, P.W. Klamut, and M. Kazimierski (Springer Verlag, Berlin, 1996).

[36] W. Belzig, C. Bruder, and M. Sigrist, Phys. Rev. Lett. 80, 4285 (1998).

[37] M. Fogelström, D. Rainer, and J.A. Sauls, Phys. Rev. Lett. 79, 281 (1997).

[38] M. Covington et al., Phys. Rev. Lett. 79, 277 (1997).

[39] M. Randeria, J.M. Duan, and L.Y. Shieh, Phys. Rev. B 41, 327 (1990).

[40] R. Haussmann, Z. Phys. B 91, 291 (1993).

[41] E. Fradkin, Field Theories of Condensed Matter Systems (Addison-Wesley, Redwood City, California, 1991).

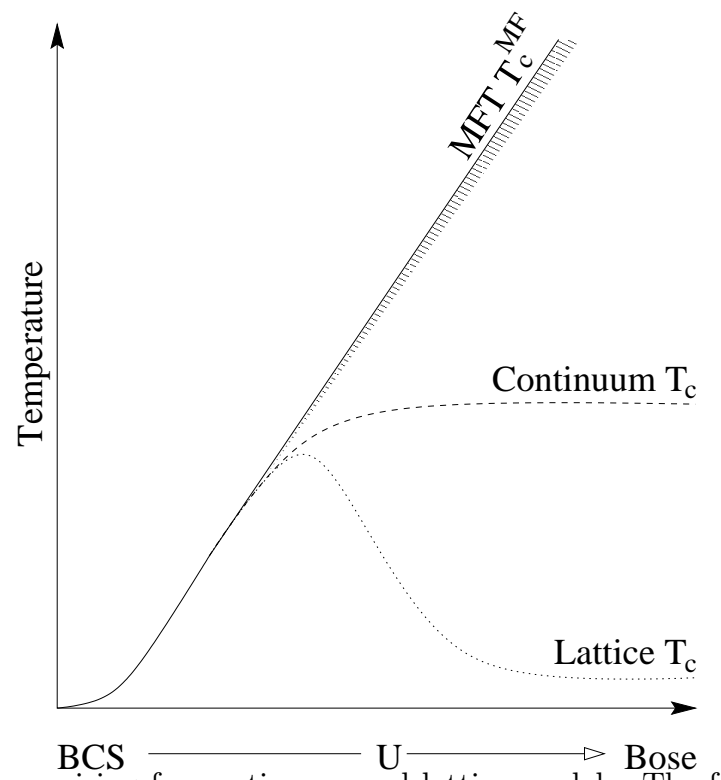

FIG. 1. Crossover from BCS to Bose pairing for continuum and lattice models. The full line shows the BCS mean field $\mathrm{T}_{c}$ evaluated for all strengths of the coupling, $U$. The broken lines show the effects of fluctuations on $\mathrm{T}_{c}$.

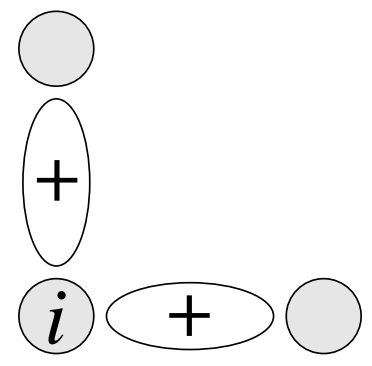

$s$ - wave

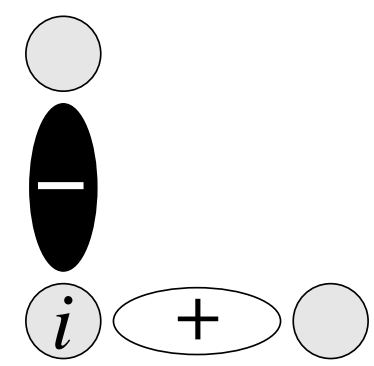

$d$ - wave 
FIG. 2. $s$ - and $d$-wave order parameters.

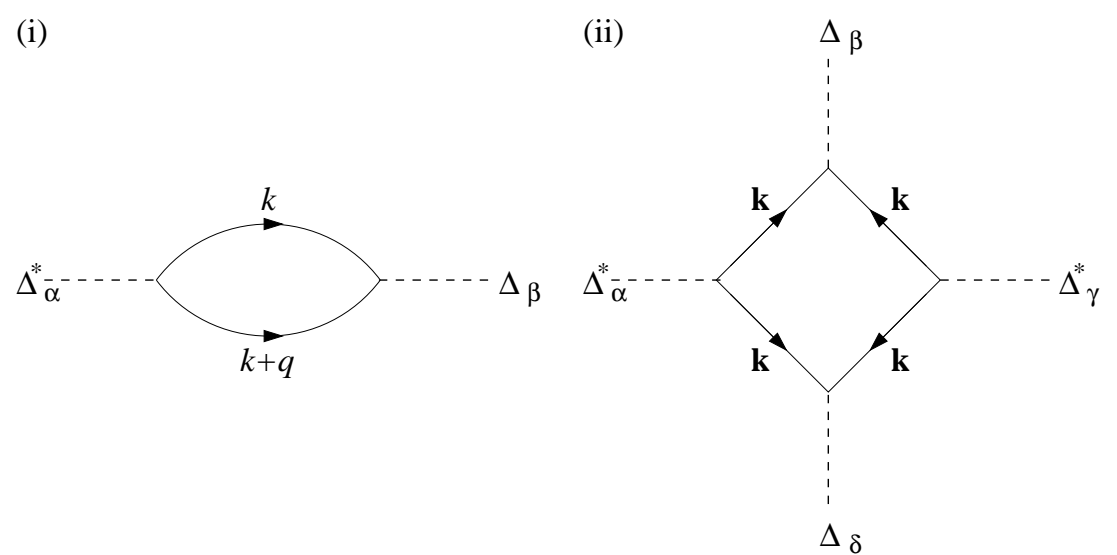

FIG. 3. (i) Quadratic and (ii) quartic terms in the expansion of $\operatorname{Tr} \ln \left(\mathbf{1}-\mathbf{V G}_{0}\right)$.

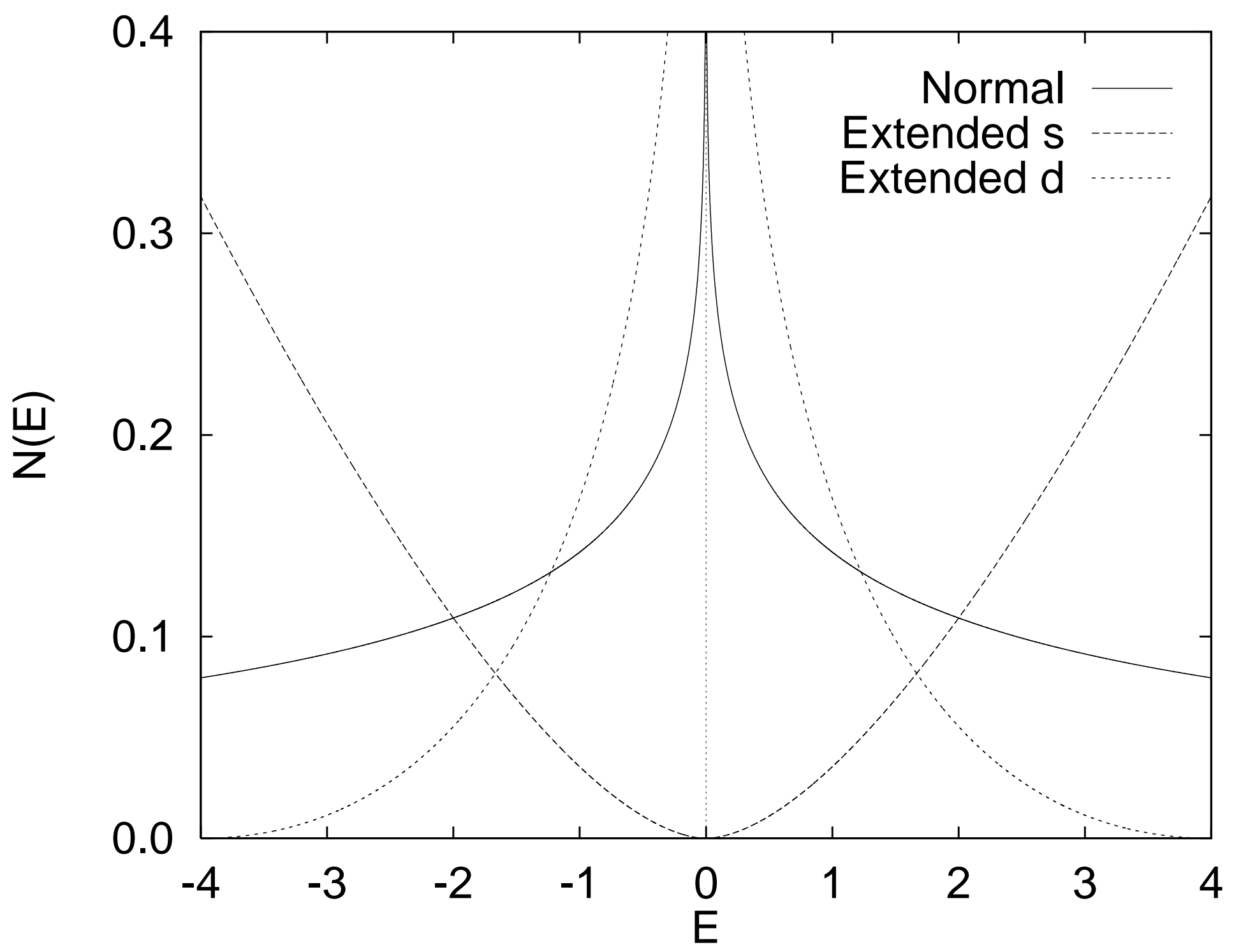


FIG. 4. Weighted densities of states (WDOS) above $\mathrm{T}_{c}$ for the normal state and the extended $s$ - and $d$-wave states.
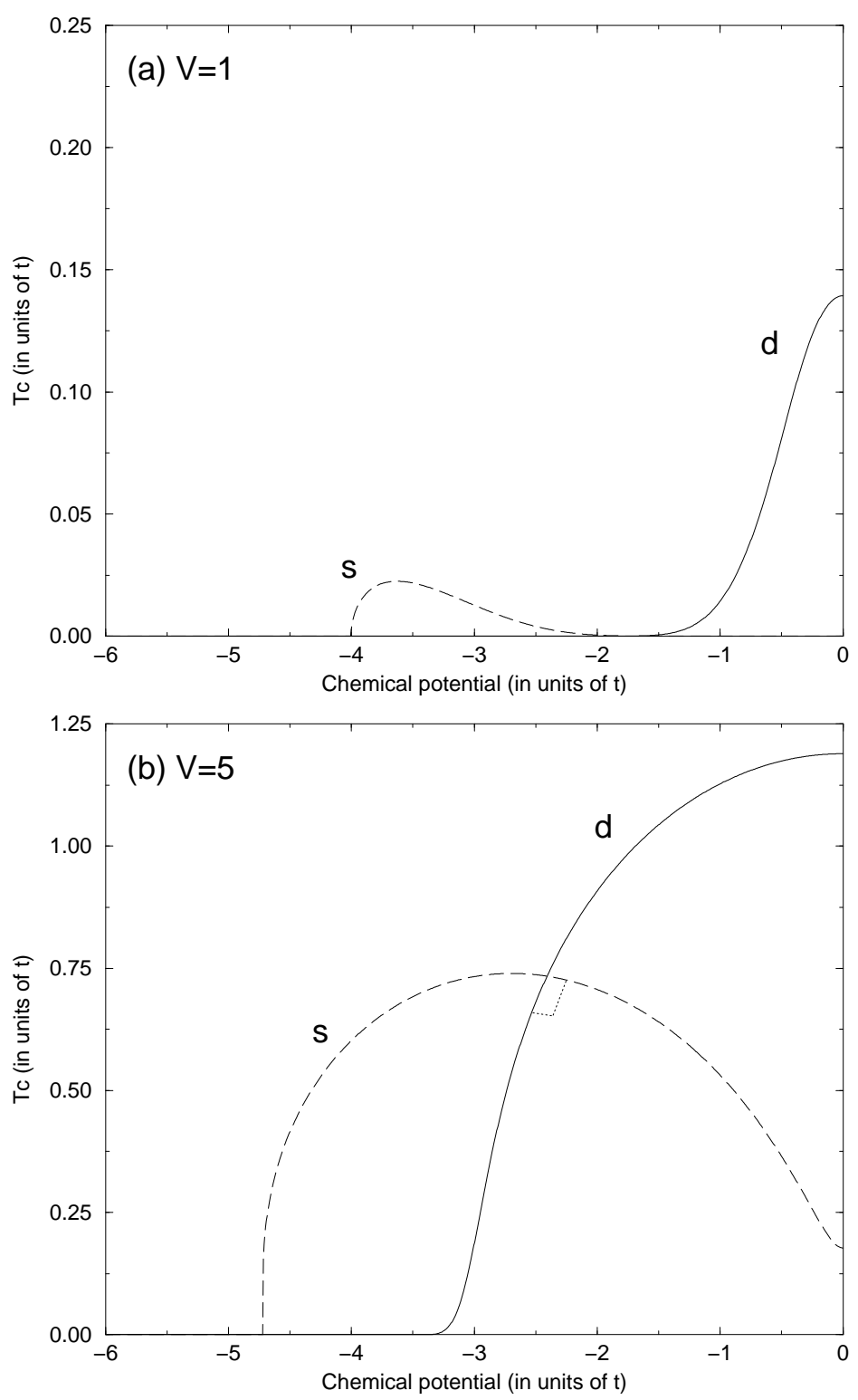


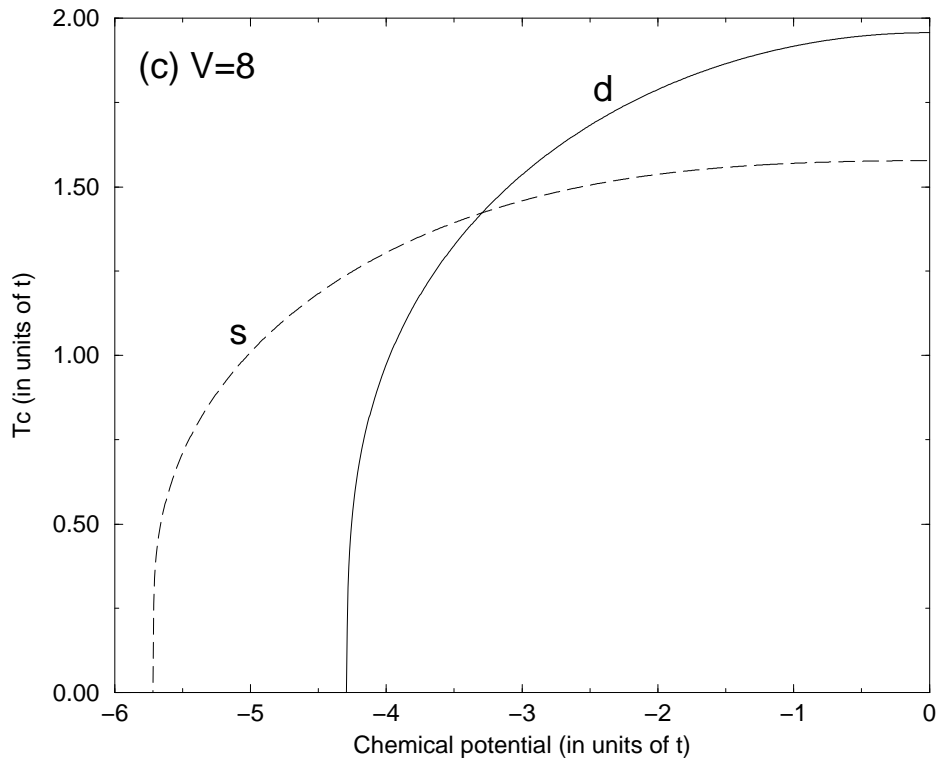

FIG. 5. Mean field critical temperature, $\mathrm{T}_{c}$, versus chemical potential, $\mu$, for $s$ - and $d$-wave superconductors with (a) $V=1 t$, (b) $V=5 t$ and (c) $V=8 t$. 


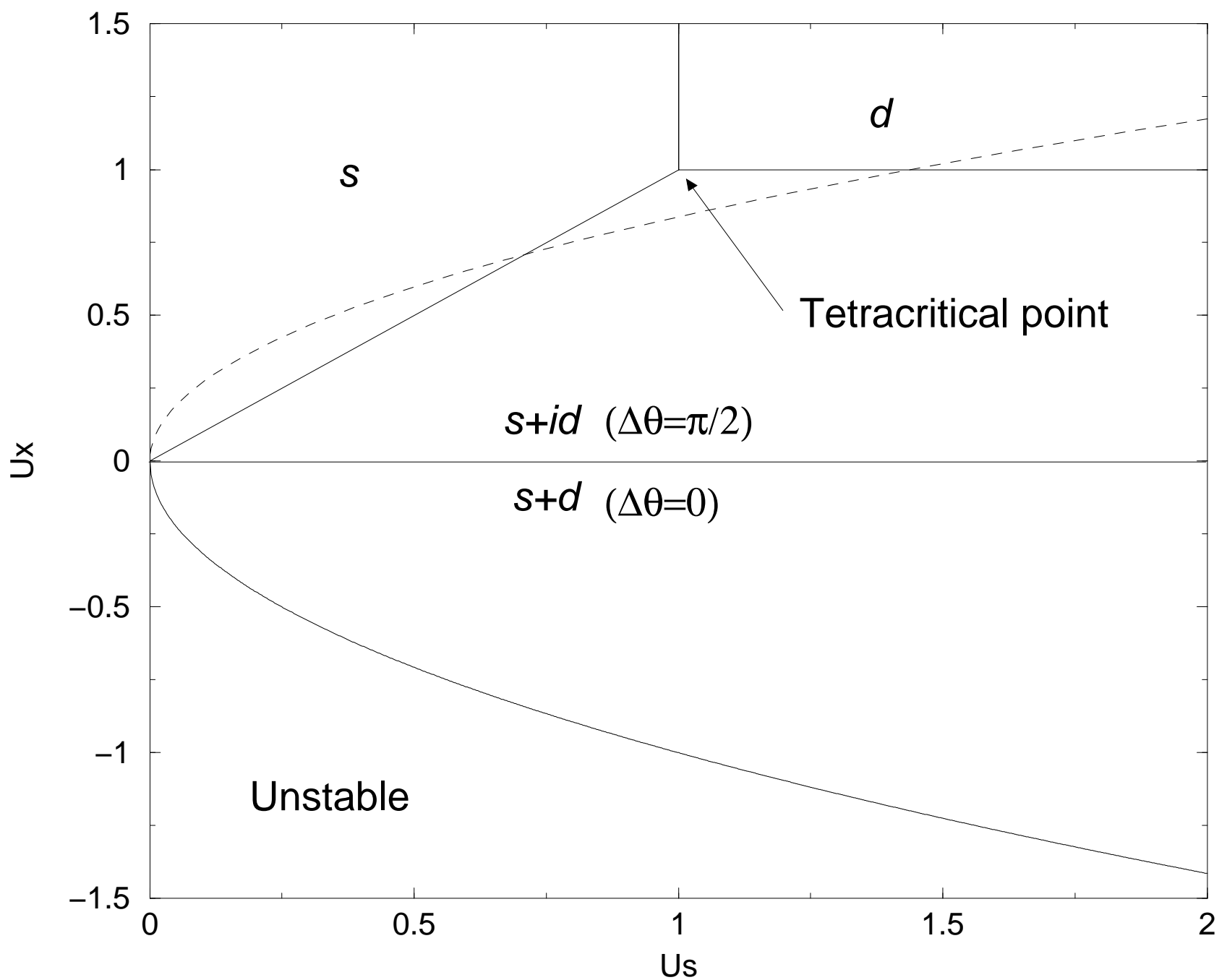

FIG. 6. Plot of the stable phases of the Landau free energy as a function of the two dimensionless parameters, $U_{s}$ and $U_{x}$. The dashed line shows parameters calculated from extended Hubbard model parameters near the tetracritical point. 

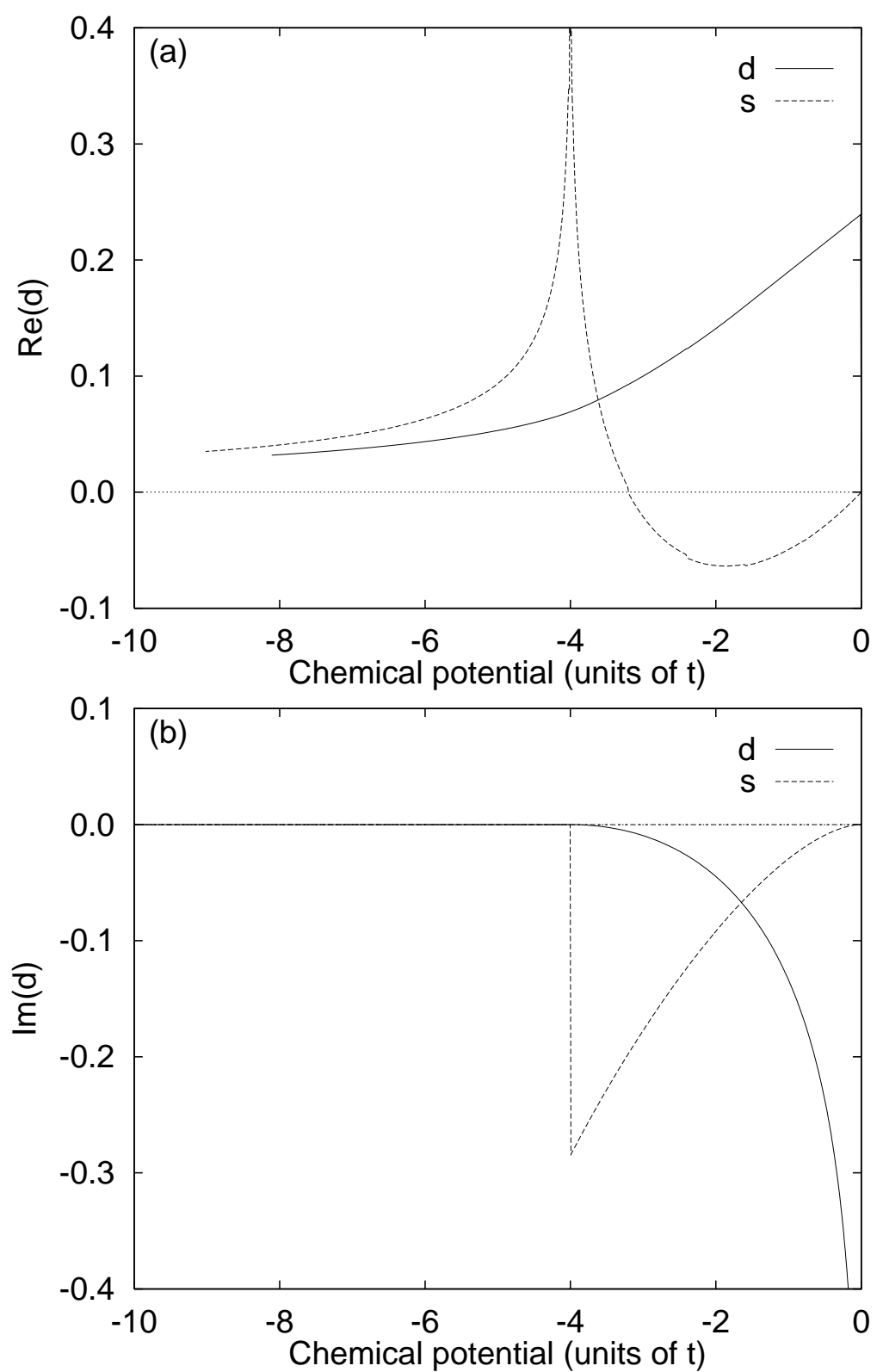

FIG. 7. (a) Real and (b) imaginary parts of the frequency coefficient, $d\left(\mathrm{~T}_{c}, \mu\right)$, for extended $s$ - and $d$-wave superconductivity at $V=16 t$.
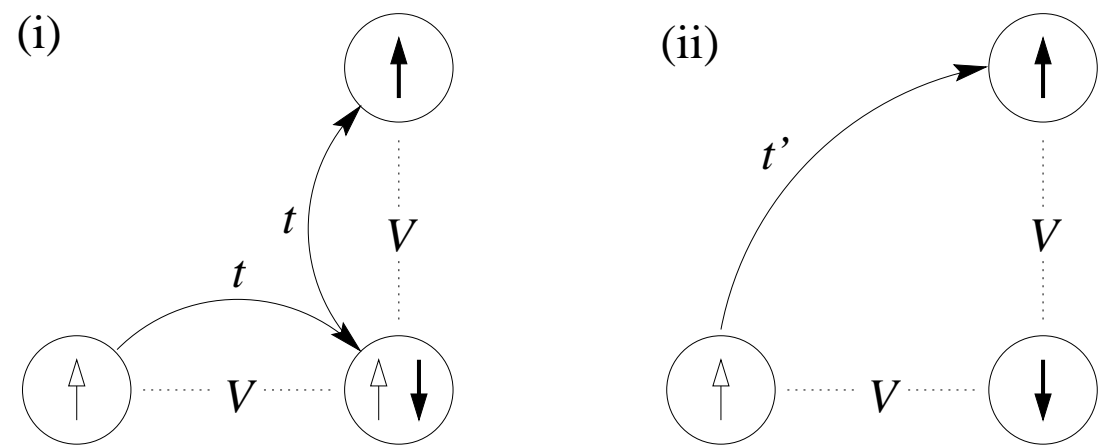

FIG. 8. Pair hopping in a tight binding model via (i) a two hops nearest neighbour process, and (ii) a one hop next nearest neighbour process. 


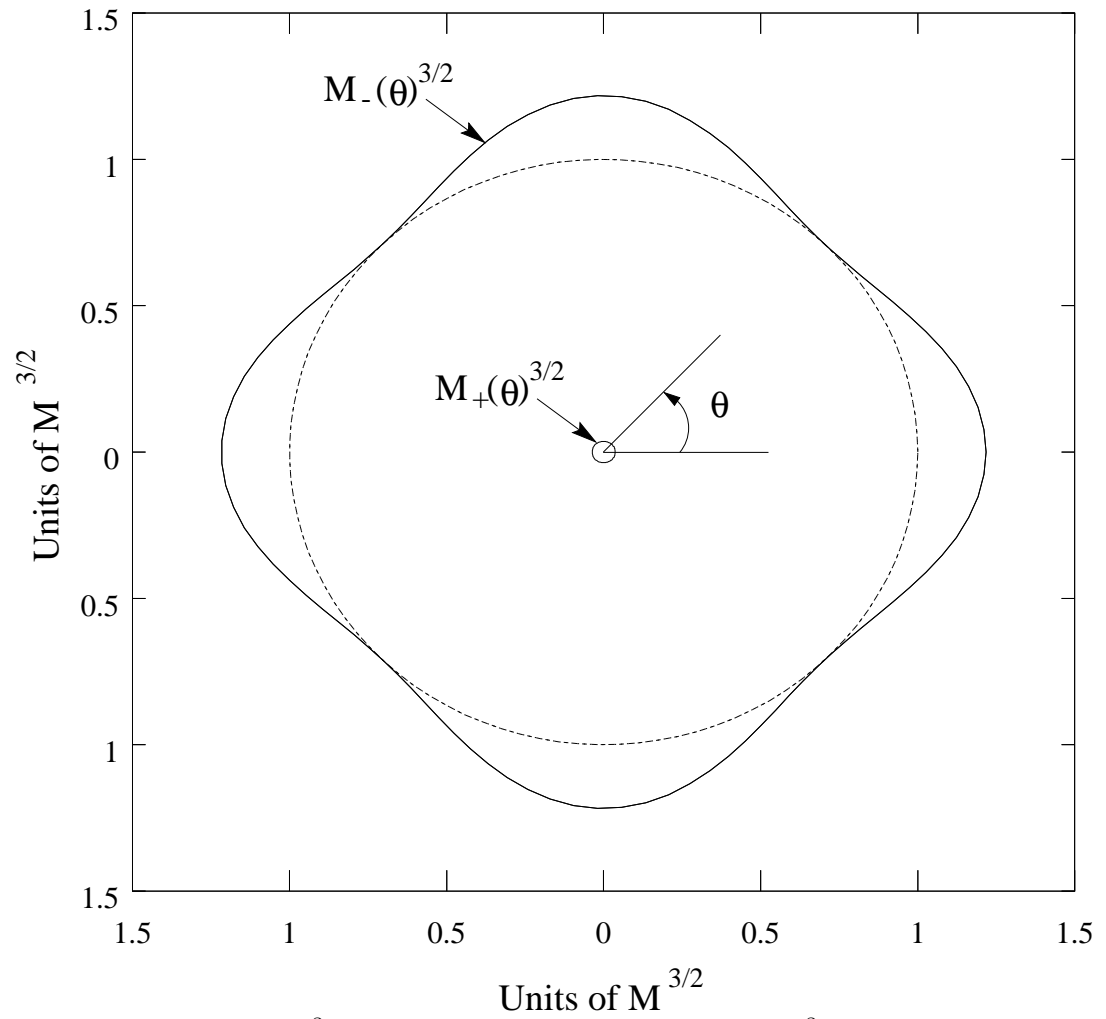

FIG. 9. Polar plot of $M_{ \pm}(\theta)^{\frac{3}{2}} \sim n_{ \pm}(\theta)$. The unit circle is $M(\theta)^{\frac{3}{2}}$ for the pure $d$-wave state.

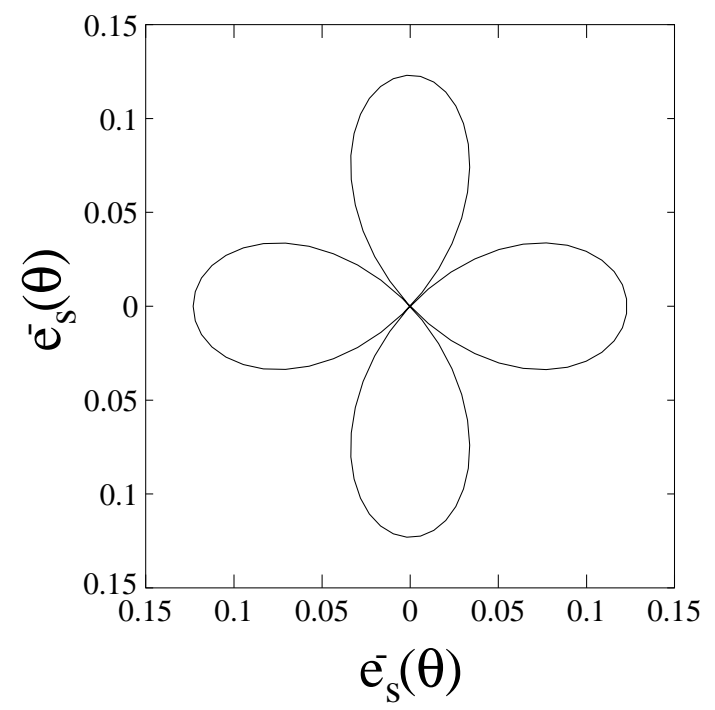

FIG. 10. Polar plot of the $s$-wave component, $\mathrm{e}_{s}^{-}$, of the dominant eigenvector. 